\title{
Methods, potentials, and limitations of gene delivery to regenerate central nervous system cells
}

\author{
Arvind Kumar' \\ Tryambak D Singh ${ }^{2}$ \\ Santosh K Singh ${ }^{3}$ \\ Satya Prakash ${ }^{4}$ \\ 'School of Biotechnology, Faculty \\ of Science; ${ }^{2}$ Department of Medicinal \\ Chemistry, Institute of Medical \\ Sciences; ${ }^{3}$ Centre of Experimental \\ Medicine and Surgery, Institute \\ of Medical Sciences, Banaras Hindu \\ University, Varanasi, India; ${ }^{4}$ Biomedical \\ Technology and Cell Therapy Research \\ Laboratory, Department of Biomedical \\ Engineering and Physiology, Artificial \\ Cells and Organs Research Centre, \\ Faculty of Medicine, McGill University, \\ Montreal, Québec, Canada
}

Correspondence: Satya Prakash Biomedical Technology and Cell Therapy Research Laboratory, Department of Biomedical Engineering, Faculty of Medicine, McGill University, 3775 University Street, Montreal, Québec, H3A 2B4, Canada

Fax + I 514398746 I

Email satya.prakash@mcgill.ca

\begin{abstract}
This review evaluates methods, success and limitations of transgenes delivery in central nervous system (CNS). Both viral and nonviral (such as liposome mediated) methods, expression and stability of transgenes have been discussed. The controlled expression and delivery techniques of transgene at the injured or diseased sites have also been discussed. Mifepristone (RU486) and tetracycline-based switch system for controlled expression could be a very useful tool for clinical purposes. Here we emphasized the importance and consequences of viral- and nonviral-mediated transgenes transfer and therapeutic ability along with advantages of controlled expressions.
\end{abstract}

Keywords: transgenes, viral vector, nonviral vector, RU486, tetracycline, CNS

\section{Introduction}

Damaged neuronal cells in the central nervous system (CNS) could not be repaired/ regenerated, leading to a partial disability or complete paralysis due to disruption of communication between brain and body. However, new findings and developments in the gene therapy techniques related to CNS have improved the prospects for recovery to some extent. While research in this field is still in the early stage, this work could lead to the clinical applications that can help to restore lost functions in the wake of brain and spinal cord injury. Development of new viral and nonviral vectors with cell type specific, physiologically-relevant and long term transgene expression at specific site is under progress. Nonviral vector such as cationic ${ }^{1,2}$ and anionic liposome ${ }^{3}$ shows no-immune response or toxicity to host. Genetically engineered cells and direct DNA transfer ${ }^{4}$ have also shown potential in certain experimental paradigms. This review provides an update and recent advancement in the gene therapy techniques related to CNS diseases and injuries.

\section{Potentials of therapeutic genes in CNS injury and disorders}

Many therapeutics genes, such as nerve growth factor (NGF), brain-derived neurotrophic factor (BDNF), B-cell lymphoma-2 (BCL-2), heat shock protein (HSP), etc, could be used to prevent and cure CNS related injuries or disorders (Table 1).

\section{NGF gene for therapeutic applications}

Nerve growth factor (NGF) is the prototypical neurotrophic factor having ability to protect peripheral sensory and sympathetic neurons from programmed cell death (apoptosis). ${ }^{5}$ Studies done so far have shown that direct CNS administration of recombinant growth factors including NGF can rescue damaged neurons and promote regeneration. ${ }^{2,6}$ In addition, NGF is able to protect adult sensory and sympathetic neurons against a variety of insults that include axotomy, ionophore treatment, exposure to hydrogen peroxide and excitatory amino acids. ${ }^{78}$ Localized diffusion of gene products into targeted region of CNS parenchyma could secrete proteins only within CNS regions relevant to neuropathological states, thus limiting the peripheral side effects. ${ }^{9}$ The intracerebroventricular 
Table I Therapeutic applications of various genes in central nervous system diseases and their current status

\begin{tabular}{llll}
\hline S No. & Gene types & Diseases & Current status
\end{tabular}

Abbreviations: BCL-2, B-cell lymphoma-2; BDNF, brain-derived neurotrophic factor; HSP, heat shock protein; IL-Ira, interleukin-Ira; NGF, nerve growth factor.

injection of NGF gene increases mNGF (messenger RNA of NGF) levels in the hippocampus that causes increased cholinergic neurotransmitter synthetic enzyme choline acetyltransferase (ChAT) activity within the brain. ${ }^{9}$ Chronic intraventricular injection of rhNGF (recombinant human Nerve growth factor) via cannulae for 21 days increased synaptosomal high affinity choline uptake, choline acetyltransferase activity, and $\left[{ }^{3} \mathrm{H}\right]$ acetylcholine synthesis by $50 \%-90 \%$ compared to lesion control values..$^{10}$ Cholinergic neurotransmission deficits after traumatic brain injury (TBI) might be the result of presynaptic alterations in the storage and release of acetylcholine (Ach) or due to conformational changes in the receptors for Ach. ${ }^{11}$

\section{BDNF gene for therapeutic applications}

Brain-derived neurotrophic factor (BDNF) is well documented for their therapeutic role in the development and survival of injured CNS. BDNF is known to stimulate axon outgrowth, branching, proliferation, differentiation, and can work as neurotransmitter. ${ }^{12}$ BDNF have also been implicated in synaptic plasticity, ${ }^{13}$ long-term memory, ${ }^{14}$ and expression of NMDA receptors. ${ }^{15}$ Loss of NMDA receptors is a cause of memory impairment ${ }^{16,17}$ after injury. ${ }^{18,19}$ BDNF is found in soluble form and induces differentiation and survival of neurons by binding to its receptor known as trkB. TrkB receptors are present in three isoforms, namely full length isoform TK+, and two truncated isoforms, TKT1 and TKT2, in the cellular membrane of mammalian CNS. In fact, trkB is a part of tyrosin kinase receptor group ${ }^{20}$ capable of adding a phosphate group at tyrosin/serine/theronine residues on target proteins after interaction with BDNF. While trkB kinase is activated by BDNF, NT-3, and NT-4, but the other subtypes
trkA and trkC are activated by NGF and NT-3, respectively. The cationic lipid-mediated BDNF gene transfection in primary hippocampal cell cultures enhances recovery of neurofilament loss produced by CNS injury. ${ }^{1}$ BDNF gene transfection could increases phosphoinositide 3-kinases (PI3-kinases) activity in CNS cells. PI-3 kinases are family of related enzymes capable of phosphorylating the 3-position hydroxyl group of the inositol ring of phosphatidylinositol. PI-3 kinases play important role in a variety of cellular responses such as mitogenesis, membrane trafficking and preventor of apoptosis. ${ }^{21}$ Moreover, BDNF has been shown to induce anti-apoptotic mechanisms after stroke that reduces infarct size and secondary neuronal cell death. BDNF is also a potent stimulator of adult neurogenesis. ${ }^{22}$ Apart from that BDNF is able to protect the brain from inflammatory brain injury in bacterial meningitis. ${ }^{23}$ Increasing the level of BDNF is an effective way to decrease mortality and to improve sequela upon bacterial meningitis.

\section{BCL-2 gene for therapeutic applications}

BCL-2 gene is known for the synthesis of anti-apoptotic protein. The name BCL-2 has been derived from B-cell lymphoma 2, and its anti-apoptotic group includes BCL-2 proper, BCL-xl, and BCL-w. Herpes simplex virus (HSV)-mediated delivery of BCL-2 gene into hippocampus and striatum in vivo can attenuate the damaging effects of ischemic brain. ${ }^{24}$ Over-expression of BCL-2 gene prevents the release of apoptosis-inducing factor. ${ }^{25}$ The post-ischemic injection of adeno-associated virus (AAV)containing BCL-2 gene has a neuroprotective effect that inhibits ischemic neuronal cell death. ${ }^{26}$ BCL-2 gene may also delay disease progression in chronic degenerative disorder such as Parkinson's disease. ${ }^{27}$ 6-Hydroxydopamine (6-OHDA) is a 
neurotoxin to dopaminergic neurons. BCL-2 produced by the vector prevented 6-OHDA-induced degeneration of neurons and increased the surviving capabilities of TH (tyrosine hydroxylase) immunoreactive neurons in the Substantia Niagra two weeks after the lesioning. ${ }^{28}$ Administration and expression of BCL-2 gene in adult rat CNS neurons prevent retrograde cell death and minimizes atrophy. ${ }^{29}$ In vivo neuroprotection of injured CNS neurons occurs by an injection of a DNA plasmid encoding the BCL-2 gene..$^{30}$ Overexpression of BCL-2 gene in primary cultured neurons protects an insult in cAMP receptor dependent manner, whereas protection is not seen against severe traumatic insults. ${ }^{31}$ These informations will provide a new insight into the molecular therapeutics for neurodegenerative conditions in future.

\section{HSP72 genes for therapeutic applications}

Heat shock protein 72 (HSP72) protein is expressed into the brain after stroke and seizures and is able to remove denatured proteins from a cell to assist new protein synthesis. Gene transfer therapy with defective HSV-vector over expressing HSP72 improves neuronal survival against focal cerebral ischemia and systemic kainic acid administration. ${ }^{32}$ Overexpression of HSP72 gene into rat brain can improve striatal and hippocampal dentate gyrus neuron survival after systemic kainic acid administration. The transgenic mice over-expresing HSP72 could attenuated hippocampal injury after focal cerebral ischemia. ${ }^{33}$

\section{Interleukin-I receptor antagonist genes for therapeutic applications}

Interleukin-1 (IL-1) acts as a cofactor and is responsible for inflammatory reaction after transient ischemia and local brain injury. Central and systemic administration of an IL-1 receptor antagonist (IL-1ra) reduces ischemic brain injury in short-term. IL-1 ra is usually produced by the normal brain cells that produce IL-1. The adenovirus vector mediated over expression of human IL-1 ra gene can attenuate ischemic inflammatory response in the mouse brain and inflammation based neuronal diseases. ${ }^{34}$ The mechanism involves binding of IL-1ra to the receptor of IL-1 preventing inflammatory reaction in ischemic cortex, striatum and corpus callosum regions. It is still unknown whether IL-1 is responsible for neuronal cell death directly or exacerbates other forms of damage or both.

\section{Ex vivo transfer mediated therapeutic applications for CNS injury and disorders}

Ex vivo gene transfer is a potential means of treating chronic neurological disorders and injury related neural degeneration.
In this approach cells are modified genetically in vitro and then transplanted to the injured site of CNS. Injured cell replacement therapy is not suitable due to the blood-brain barrier (BBB). To circumvent the BBB, ex vivo gene therapy is most acceptable and is able to traverse the $\mathrm{BBB}$ or other membranes of the CNS. Fibroblast, peripheral nerves, astrocytes, and myoblasts cells could be used for the ex vivo gene therapy in the CNS.

Fibroblast cells modified with NGF genes have been transplanted into the brain and spinal cord to provide neurotrophic factors and substrates for axonal growth and elongation. NGF secreting fibroblast cells transplant have been shown to prevent degeneration of cholinergic neurons in the basal forebrain of primates. Transplant induces sprouting of sensory, motor, and noradrenergic neurites after spinal cord injury. The controlled and targeted expression of tetracyclineregulated ex vivo delivery of NGF is possible at transplanted sites. ${ }^{34}$ Genetically transduced Schwann cells grafted to spinal cord injury sites increase axonal growth by the over expression of NGF. ${ }^{35,36}$ When fibroblasts cells, genetically modified to secrete NGF, BDNF, NT-3, and basic fibroblast growth factor (bFGF), transplanted into the central gray matter of the spinal cord in the adult rats, sensory neurites of dorsal root origin extensively penetrated NGF-, NT-3-, and bFGF-secreting grafts, whereas no growth has been found in BDNF-secreting grafts. ${ }^{37}$

Injured CNS tissues and damaged neurons are unable to regenerate their axons spontaneously. Genetically modified peripheral nerves can be implanted ex vivo, in transected sciatic nerve, avulsed ventral root, hemi-sected spinal cord, and intact brain to overexpress the transgene encoding growth promoting NT-3 proteins that improves the permissive properties of the nerves..$^{15}$ The rat fibroblasts, genetically modified to produce NT-3, grafted to acute spinal cord dorsal hemisection lesion cavities showed significant partial functional recovery in corticospinal axon growth at distal to the injury site. ${ }^{38}$

Astrocytes originated from CNS have efficient secretory mechanisms to play an important role in neuronal growth. Human adult astrocytes modified with specific transgene could be used for ex vivo gene therapy. Ex vivo cell transplantation decreases the chances of immunological rejection at minimum level and thus obviating the side effects of immunosuppressors. ${ }^{39}$

Myoblast cells ${ }^{40}$ and astrocytes ${ }^{41}$ could be genetically modified to express tyrosine hydroxylase $(\mathrm{TH})$ and dopamine in culture. These modified myoblasts, not showing immuno-rejection property, might be used as gene carriers for ex vivo gene therapy in the CNS. Thus, ex vivo gene 
therapy in the CNS could be an efficient and convenient tool for the future. ${ }^{42}$ Deficiency of beta-glucuronidase (GUSB) causes multisystem progressive degenerative syndrome, mucopolysaccharidosis (MPS) type VII (Sly disease), in adult brain that could be cured by transplanting engineered GUSB-secreting cells to super-secrete the normal enzyme for export to surrounding neural tissues. ${ }^{43}$

\section{Controlled expression of therapeutic genes in CNS}

There is a need to control the transgene expression to prevent adverse effects of overexpression. The concept of molecular switches is based on the use of tissue-specific promoters, which confers restricted expression of transgene appropriately within the tissue. Appropriate regulation means the capability of the system to turn the transgene on and off in response to symptoms (expression) of the targeted disease. Many gene switch systems are available to control transgene expression but in CNS, mifepristone (RU486), and tetracycline (tet)-based switch systems are important.

\section{Transgene regulation by inducible promoter (mifepristone)}

Transgene expression can be controlled by using a specific promoter whose activity can be controlled by mifepristone, ${ }^{44}$ a progesterone hormone antagonist. Mifepristone is a 19-nonsteroid which has a specific high affinity binding to the progesterone and glucocorticoid receptor. Mifepristoneresponsive gene switch system has become most attractive for an application in traumatized CNS. The synthetic progesterone antagonist readily crosses the BBB when administered systemically. ${ }^{45}$ In this system the transgene to be regulated is placed under transcriptional control of a promoter, which in turn is activated by a specific transactivator, consisting of a fused tripartite protein. The tripartite proteins are Gal4 (Yeast DNA-binding domain), HBD (mutated progesterone receptor that binds specifically to mifepristone), and VB 16 (activation factor derived from HSV). The vector used for gene therapy encodes the fusion protein, and either a cytomegalovirus (CMV) or tissue-specific promoter, which drives its expression after delivery. In its native state, the transactivator does not induce transgene transcription but binding of mifepristone to the transactivator enable the administered transactivator to immediately initiate the transgene expression. This switch system has been used to regulate genes systemically when transferred with either plasmid $\mathrm{DNA}^{46}$ or adenoviral vectors,${ }^{47}$ or in conjunction with HSV vector-mediated gene transfer. ${ }^{48}$ With inclusion of a mifepristone-responsive gene switch into gene delivery vector, transgene expression could be regulated according to therapeutic need.

\section{Transgene regulation by tetracycline antibiotic-based gene switch}

Tetracycline-based switch system is based on the use of inducible elements and factors along with transgene, regulated by the administration of a second-step drug or by the end product. Based on the above principle, highly controlled gene expression of recombinant Ad and AAV vectors using combinations of a tissue-specific promoter and a tetracycline transcription factor have been constructed. ${ }^{49}$ Thus, it is possible to transfer a putative therapeutic gene to specific tissue in a completely dormant state. Expression of the dormant gene can be induced by the oral administration of a second-step drug (rapamycin or mifepristone) that directs the formation of an active transcription factor complex on the silent promoter of the transferred gene. Despite the above information, no literature is available to show a switch based promoter in AAV vector for the transfer of therapeutic gene in brain injury. In contrast, a switch based promoter with stable expression of a constitutive AAV-erythropoietin vector in non-CNS tissues has been successfully demonstrated. Moreover, the regulation of tet-promoter is simple because gene induction or repression is being controlled by only one protein. Also, this switch system can be packaged into a single vector due to smaller size of tet-transactivator and tet-regulatable promoter. The minimal CMV promoter is fused to the tet-operator sequence to stimulate transcription of tet-transactivator in the absence of tetracycline. Tetregulatable gene expression system can release NGF-GFP (green fluorescent protein) in a controlled manner from primary rat fibroblasts in a dose-dependent manner by the exposure to the tet analog doxycyline. ${ }^{50}$

\section{Recent developments in CNS gene delivery vectors/carriers}

Many techniques (such as viral and nonviral vectors, chemical carriers, and physical forces) could be used for targeted delivery of transgenes at the diseased or injured CNS site (Figure 1).

\section{Viral vectors for CNS therapeutic gene delivery}

Viral vectors have become important tool for the gene delivery at particular site in the brain. Different strategies are 


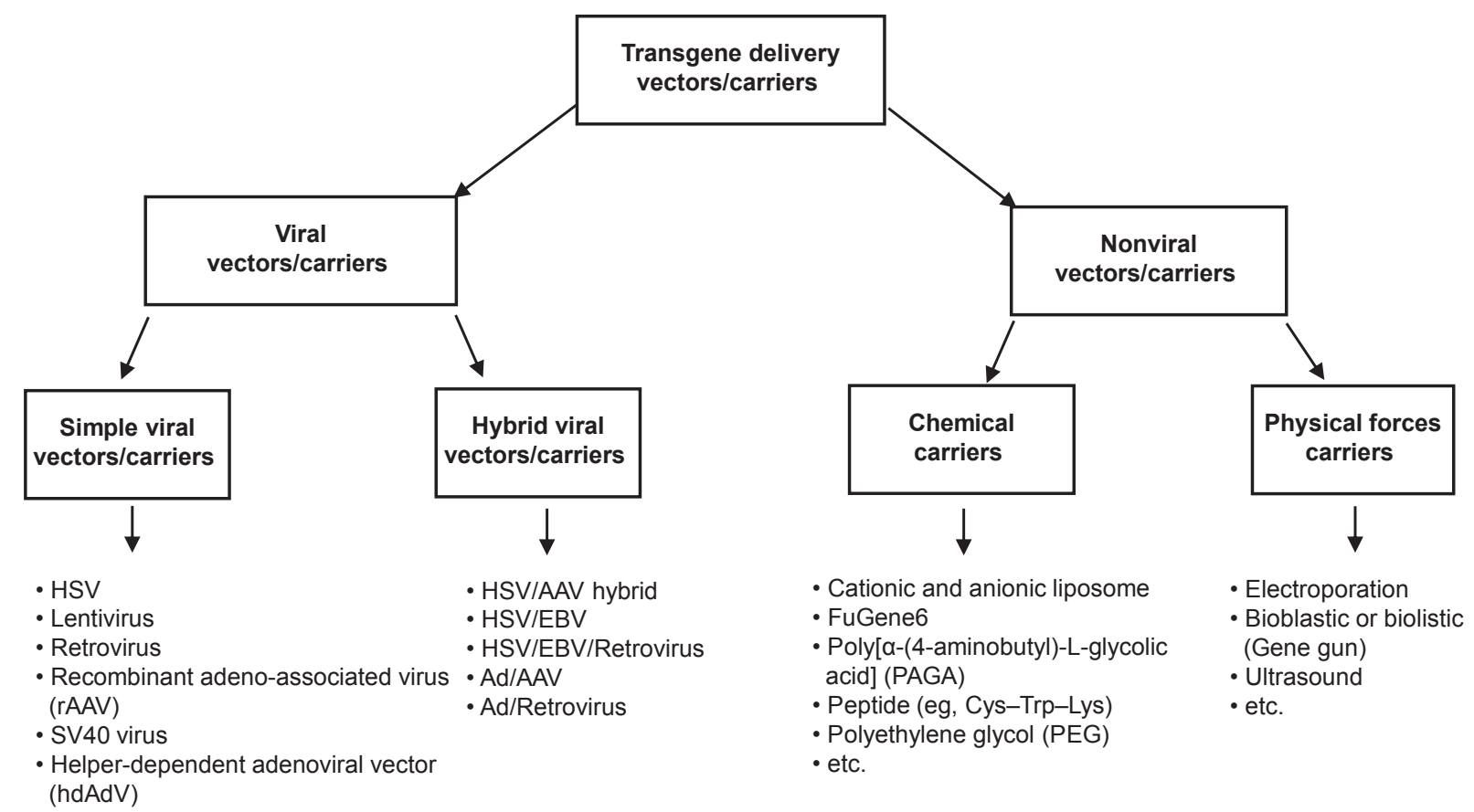

Figure I Vehicles for gene delivery in central nervous system to regenerate the damaged cells.

Abbreviations: AAV, adeno-associated virus; Ad, adenovirus; EBV, Epstein-Barr virus; HSV, herpes simplex virus; SV-40, simian virus 40.

used to deliver genes to CNS and enhance its distribution. One way is to inject the viral vectors directly into the cerebral lateral ventricles (LVs). By this way, virus will be delivered throughout the CNS. ${ }^{51}$ Another way is to inject at multiple sites to cover a large volume. Some agents such as Mannitol ${ }^{12}$ and heparin ${ }^{53}$ are used to increase the distribution of vectors. Some important viral vectors have been discussed below.

\section{Simple viral vectors}

\section{Herpes simplex virus (HSV) vectors}

HSV is a neurotrophic virus having ability to establish a life-long latent state in neurons. It is an enveloped (envelope contains at least 10 glycoproteins) double-stranded DNA encoding more than 80 identified genes bearing $152 \mathrm{~kb}$ viral genome. HSVs infect neurons by rapid retrograde axonal transport mechanism, thus providing a means of gene transfer to targeted cells that are not easy to reach directly. ${ }^{54}$ Two types of HSV-vectors were constructed for gene transfer: recombinant virus (RV) and amplicon vectors. Recombinant replication-conditional viruses contain one or more mutated genes (such as $\mathrm{r} 34.5$ or ICP6) in the full genome to reduce overall toxic effects. Replication defective $\mathrm{RV}$ vectors or multiplication-defective genomic HSV vectors were constructed by deleting all immediate-early (IE) genes such as ICP0,${ }^{55}$ ICP4, ICP22, ICP27, and ICP47 76,57 that encode transactivating factors. Such deletion eliminated expression of other viral genes ${ }^{56,58}$ that may be toxic to cells. These multiple gene-deleted vectors have many advantages: (a) can propagate to high titers in the complementing cell lines, and (b) long-term expression of latency associated transcripts (LATs) in genome does not depend on IE-genes. This provided a chance to construct a highly defective HSVvector that can readily persist in a latent state in neurons, and transgenes could be expressed using the LAT promoter. Basically HSV amplicon based vectors are plasmid-based DNA constructs. A typical HSV amplicon construct contains, in addition to the gene of interest, a copy of HSV replication origin (oris) and packaging signal (pac). When these vectors were introduced into cells together with a helper HSV, the amplicon plasmid DNA efficiently amplified and packaged into viral particles. Helper virus free amplicon stock could be generated by co-transfecting amplicon set of cosmids or BAC plasmids containing fragmented or modified HSV genome. ${ }^{59}$

The HSV amplicon mediated gene transfer has many advantages over recombinant HSV vectors. These advantages include nontoxic and nonimmunogenic responses to the target cells, since amplicon based vectors do not encode any viral gene products. Amplicon is a multiple gene delivery system because each amplicon contains 10-15 copies of the inserted gene due to its concatemeric form. This gives much higher expression of transgene in comparison to recombinant virus 
containing similar type of gene cassette.$^{60}$ Moreover, it is easy to insert gene of choice into amplicon vectors that has long-term transgene expression in different areas of CNS. ${ }^{61}$ In order to improve the proportion of amplicons generated, a selection system for amplicon production is developed in which the HSV thymidine kinase (TK) gene is inserted into an amplicon plasmid and an HSV mutant with both TK and glycoprotein $\mathrm{H}(\mathrm{gH})$ genes deleted is used as a helper. ${ }^{60} \mathrm{HSV}$ virus has been designed in which the prokaryotic Cre-loxP site-specific recombination system is employed. In this system, gH-helper virus is engineered in such a way that loxP sites flank both copies of its packaging signals and thus generated stocks with high amplicon titer and much improved amplicon over helper virus ratio. The injection and expression of HSV vectors containing $\beta$-NGF gene under the transcriptional control of either human cytomegalovirus immediate early promoter (HCMV Iep) element or HSP-latency active promoter (HSV-LAP2) produced biologically active NGF in transfected PC-12 cells. HSV virus-mediated NGF synthesis induces expression of superoxide dismutase and catalase, and is effective in protecting cells from apoptosis induced by hydrogen peroxide. ${ }^{62}$ Replication defective genomic HSV vector mediated transfer of $\beta$-NGF, under the control of either the LAP2 or HCMV Iep promoter, into the knee joint of animals has been effective for treatment of peripheral neuropathesis. ${ }^{57}$ Amplicon HSV has a lot of advantages but it still has some limitations because it is usually difficult to generate a stock with a high amplicon titer and high ratio of amplicon to helper virus.

\section{Lentivirus vectors}

Lentivirus vectors are derived either from the HIV-1 (human immunodeficiency virus type-1) vector or FIV (feline immunodeficiency virus) vector after genetic manipulation ${ }^{63,64}$ and able to carry $8 \mathrm{~kb}$ of sequence to any neuronal cell type with sustained expression in which normal cellular functions are not compromised either in vitro or in vivo. ${ }^{65} \mathrm{HIV}$-1-derived vectors have ability to integrate into the host genome of dividing and nondividing cells, and hence can be utilized for the transfer of genes with stable expression even in post-mitotic neurons. Lentiviral vectorencoded beta-galactosidase transgene showed very efficient transfer, integration, and sustained long-term expression without showing any pathology in adult rat brains. In vivo gene transfer using lentiviral vector depends on a functional integrase protein. ${ }^{66} \mathrm{~A}$ recent report suggests that lentiviral vectors surpass retroviral vectors in efficient long-term and stable gene transfer in adult neural stem cells. ${ }^{67}$ On the other hand, the HIV-1 has a broad host range and can infect brain, liver, and muscle cells. The targeted transduction of transgene in the CNS was achieved using specific envelope glycoproteins to pseudotype lentivrial vectors. The use of Ebola-pseudotyped virus, Mokola-pseudotyped, and murine leukemia virus (MuLV)-pseudotyped lentiviral vectors are more efficient and stable alternatives to vesicular stomatitis virus glycoprotein (VSV-G)-pseudotyped vector gene for the transduction of transgene in mouse CNS. ${ }^{68}$ Despite of these developments the clinical application of both the HIV-1 and FIV vectors for CNS has yet to be confirmed experimentally.

\section{Retrovirus vectors}

The higher and unequal efficiency of retroviral vectors to integrate their genome into host cell chromosomal DNA has made it the first choice for many gene therapy applications. In many clinical trials so far no single case has been reported which attributes adverse events of insertional mutagenesis, caused by retrovirus vector application. The vectors have $8.5 \mathrm{~kb}$ of transgenes flanked by retroviral long terminal repeat (LTR) regions, a virion packaging signal, and a primer binding site for reverse transcription. After delivery into cells, double-stranded DNA sequences can be reversely transcribed which can then get integrated randomly into host cell genome. This vector has limited use for gene delivery to CNS because of their ability to transfer genes only to dividing cells, yet have been well suited for on-site delivery to neural precursors for lineage studies. ${ }^{69}$

\section{Recombinant adeno-associated viral ( $\mathrm{rAAV}$ ) vectors}

There are 11 AAV serotypes have been reported so far. ${ }^{70-72}$ They infect cells from several diverse tissue types. Capsid serotype is the main determinant of tissue specificity and pseudotyping of AAV vectors to alter their tropism range for their use in gene therapy. Different serotypes can bind to different cellular receptors. Among these, serotype 2, 4, 5 (AAV2, 4, 5) have been studied most extensively ${ }^{73,74}$ and are found efficient for transduction in the mammalian brain. ${ }^{73}$ rAAV vector is the vector of choice for gene delivery to neurons due to several advantages: (a) easy to manipulate genetically, (b) ability to transduce most tissues including terminally differentiated cells, (c) purification to high titers, and are (d) relatively safe. The replication defective recombinant adenoviruses are commonly used as gene transfer vectors because of their less immunogenicity and ability to transduce both neurons and glial cells efficiently. ${ }^{75} \mathrm{rAAV}-$ mediated in vivo gene transfer has demonstrated efficient 
long-term transduction (from three months to more than 1.5 years), lack of cytotoxicity and cellular immune response in target tissues, especially in the CNS. ${ }^{76}$

The contamination risk could be eliminated in rAAV production by (i) substituting adenovirus with a plasmidbearing E2a, E4orf6, and VA helper function, and (ii) growing on HEK 293 cells which express Ad E1a and E1b. ${ }^{77,78}$ There are two types of toxicity due to (a) aggregation of rAAV with cell lysate proteins, and (b) residual hyperosmotic $\mathrm{CsCl}_{2}$ during purification step. ${ }^{79}$ Both types of toxicity could be removed by substitution of density centrifugation with isoosmotic and inert medium iodixanol. Affinity chromatography is being used for high level of purification of rAAV. ${ }^{80,81}$ The heparin sulfate proteoglycan (a cellular receptor for attachment and infectivity of $\mathrm{AAV}-2^{81}$ ) and virion-specific monoclonal antibodies ${ }^{82}$ could be utilized as core facility for the purification and production of rAAV using ligand specific matrix chromatography. Moreover, rAAVs have been developed having capability to express human protooncogene BCL-236 that confers an ability to block neuronal death after transient ischemia. rAAVs have capability to package $6.6 \mathrm{~kb}$ vector sequence that could be used for gene therapy for hemophilia A and other diseases with large cDNA such as muscular dystrophy and cystic fibrosis. ${ }^{83}$

Aromatic L-amino acid decarboxylase (AADC) converts L-dopa to dopamine. The AADC gene encoded in rAAV vectors has been used for therapy purposes to treat Parkinson's disease. ${ }^{84}$ Prevention of dopaminergic neuron death by AAV vector-mediated glial cell derived neurotrophic factor (GDNF) gene transfer in rat mesencephalic cells has also been reported.$^{85}$ The behavioral recovery in 5-hydroxydopamine-lesioned rats by co-transduction of striatum with tyrosine hydroxylase and AADC genes using two separate AAV vectors is possible. ${ }^{85}$ Although, rAAV has shown its potential in gene therapy, further improvements are needed to consider for clinical uses.

\section{Simian virus 40 (SV40) vectors}

SV40 virus is a member of the nonenveloped particle of polyoma family of viruses with double stranded circular DNA genome of $5.25 \mathrm{~kb}$. SV40-derived vectors can express both in vitro or in vivo with long term transgene expression either into caudate-putamen or lateral ventricle after injection. ${ }^{86}$ For transgene expression in CNS, rAAV utility is measured in comparison to SV40. SV40 can package genomes up to $5.7 \mathrm{~kb}$ without difficulty after deleting structural genes. rSV40s (recombinant SV40) do not elicit detectable neutralizing antibody responses. ${ }^{87}$

\section{Helper-dependent adenoviral vectors}

Third generation adenovirus vectors (AdV), called gutless or helper dependent adenoviral vector (hdAdV), have been developed that retain only the sequences necessary for packaging and replication of viral genome and lack all structural genes ${ }^{75}$ thus extending the cloning capacity of the vector (up to $37 \mathrm{~kb}$ ). This novel construct has capacity to propagate to high titers without contaminating helper Ad virus using a Cre-lox-based recombinase system. Gene transfer by hdAdV demonstrated persistent gene expression with negligible toxicity in peripheral organs such as liver ${ }^{88,89}$ hdAdV has been used to transduce genes to CNS for stable gene transfer that significantly prolonged transgene expression (up to 183 days). ${ }^{89}$ The number of macrophages and $\mathrm{T}$ lymphocytes infiltrating the brain could be greatly reduced in hdAdVtreated host in comparison to first generation adenovirus (fgAd)-treated host. ${ }^{6}$ The hdAdV provides equally efficient or higher infectivity but significantly reduced toxicity than fgAd vectors. ${ }^{90}$ Low toxicity is extremely important for the clinical applications of hdAdV as a future tool for gene delivery to CNS. Studies delivering $\alpha$-antitrypsin and leptin gene using hdAdVs in vitro or in vivo have shown that these fully deleted Ad vectors can provide high-level, long-term gene expression with improved tolerance due to absence of the viral genome. ${ }^{88,89}$ However, hdAdV infection causes moderate but significant changes in cell function and viability at excessive viral titers in primary neuronal cultures. ${ }^{91}$

The expected improvements for clinical use of hdAdVs are: (1) to remove all helper viruses from the hdAdV preparation to avoid even trace amount of contamination, (2) development of targeted hdAdVs to localize gene transfer to specific cell types, (3) production of high-titer vector without the cost of contamination by wild-type AAV, and (4) use of molecular switches for controlled expression of transgene by the vectors. Moreover, it is possible that some of improvements can be achieved through the construction of hybrid viral vectors utilizing two different viruses.

\section{Hybrid viral vectors}

These vectors are also known as chimeric or hybrid viral vectors and constructed or developed to achieve reproducible and stable gene delivery to the CNS or other parts of the body. Such kind of hybrid vectors has been constructed to incorporate different viral elements with particular features to stabilize the transgenes at transfected site. Various types of vectors have been constructed by utilizing the combination of two or more viral elements or gene sources. Some important hybrid viral vectors have been discussed below. 


\section{HSVIAAV hybrid amplicon vectors}

The hybrid amplicon vectors contain oris and pac signals of HSV-1 and ITR sequences of AAV to flank the transgene. It was produced either with or without the AAV rep gene to evaluate its importance in producing sustained transgene expression in human glioma cells. ${ }^{92}$ The hybrid amplicon vector extended transgene expression in dividing human glioma cells well beyond the capacity of HSV amplicons. Higher transduction efficiency in primary neuronal cultures and longer expression of the transgene in neurons were also noted in hybrid amplicon-mediated gene transfer in comparison to AAV and AdV. ${ }^{93,94}$

\section{HSV/EBV and HSV/EBV/retrovirus hybrid amplicon vectors}

Amplicon elements of HSV, latent origin of DNA replication (ori-P), and Epstein-Barr nuclear antigen-1 gene (EBNA-1) elements of Epstein-Barr virus (EBV) are used to construct HSV/EBV hybrid vector. Inclusion of ori-P and EBNA-1 increased the stability of transgene during replication in the dividing cells..$^{95} \mathrm{~A}$ hybrid vector having the character of HSV and retrovirus has also been constructed..$^{96,97}$ This hybrid $\mathrm{HSV} /$ retrovirus vector confers ability of retrovirus vector to transduce into both dividing and nondividing cells in a single step of infection. Since this new construct has ability to transduce into nondividing cells it can be efficiently utilized for the transfer of gene in CNS.

\section{Ad/AAV hybrid vectors}

This hybrid vector encodes the AAV Rep78 protein and an ITR-flanked transgene. ${ }^{98}$ Another type of Ad/AAV vector consist of an AAV ITR-flanked transgene in which the AAV Rep isoforms are conjugated to the Ad-virion via poly-lysine bridge to site specific delivery of the transgene.

\section{Ad/retrovirus hybrid vectors}

This chimeric vector is constructed to increase the transfection capability and to successful expression of transgene in the neighboring cells. When any cell is transfected by the hybrid (Ad/retrovirus) vector, transfected cells produce two types of functional progeny: a retroviral packaging functions and retroviral vector/transgene sequences. The progeny after release can infect neighboring cells leading to the incorporation of transgene. ${ }^{91}$

\section{Nonviral vectors for CNS therapeutic gene delivery}

Problems associated with viral vector gene delivery systems (eg, unwanted deleterious immune response or changes in the properties of delivered virus due to endogenous recom- bination and mutagenic behavior leading to oncogenesis) lead to the development of nonviral vector delivery systems. This contains use of chemical carriers and naked gene delivery using electro-poration, gene gun (bio-ballistic or biolistic), ultrasound and hydrodynamics (high pressure).

\section{Chemical carriers mediated CNS gene delivery}

Chemical carriers are designed to protect the delivered DNA from nuclease activity. Nonviral vector such as cationic ${ }^{1}$ and anionic liposome ${ }^{3}$ with no immune response or toxicity have been reported. Although, the cationic liposome mediated gene transfer to different cell types is successful, this method is limited in use due to its lower transfection efficiency in compared to viral systems. A novel compound, FuGene6, ${ }^{99}$ has also been tested to transfer gene of choice using reporter plasmid pEF-beta galactosidase. This compound has less toxicity in comparison to the Lipofectamine, as shown by Trypane blue staining.

The novel compound FuGene6, a commercially-available cationic lipid, has a very high potential to transfer DNA into cells of glial origin, and might be an interesting candidate for ex vivo and in vivo gene therapeutic approaches. ${ }^{99}$ The FuGene6-mediated gene transfer is useful to transfer the reporter gene $\beta$-galactosidase into C6 glioma cells, primary glia, and primary neurons. ${ }^{100}$ The cationic liposome DNA complexes (CLDCs) produces significant levels of expression of both reporter genes and biologically relevant genes in nonparenchymal cells lining CNS. ${ }^{9}$ The intracerebroventricular or intrathecal injection of either CLDCs containing the $\beta$-galactosidase ( $\beta$-Gal) gene produced patchy and widely scattered areas of $\beta$-Gal expression. The chloramphenicol acetyl transferase (CAT) reporter gene product is present at significant levels after single intracerebroventricular injection. To improve efficiency, the fusion proteins derived from the Sendai virus is incorporated into cationic liposomes to avoid degradation by endocytosis. ${ }^{101}$ Nonhistone chromatin proteins, a high mobility group protein, have been incorporated into the liposome for the transfer of transgene at specific site. ${ }^{102}$ Many cationic polypeptides eg, polylysine, spermidine, etc having capacity to bind the negatively charged DNA are used to target the gene transfer to the cell linked with specific ligands. Although liposome mediated gene transfer into the brain has nontoxic and nonimmunogenic effect, it has low efficiency of transgene expression in compared to the viral mediated transgene expression. The ori-P and EBNA-1 gene elements from the EBV have been used in the liposome-associated DNA to prolong the stability of the 
transgene in the dividing cells. ${ }^{103}$ Biodegradable polymer, poly[ $\alpha$-(4-aminobutyl)-L-glycolic acid] (PAGA) a derivative of poly-L-lysine is under trial for delivery of transgene. Other than PAGA, PEG (polyethylene glycol) and peptide mediated (eg, Cys-Trp-Lys) are also under trial.

\section{Physical forces-mediated CNS transgenes delivery}

Successful transfer of naked DNA into the adult mouse brain has been reported. Microprocessor-controlled injector, an important tool for nonviral gene transfer technique, has successfully been used to deliver the gene into the CNS. ${ }^{40}$ Reports are also available to show the use of electroporation technique to deliver naked transgene in the skin at particular site. ${ }^{104,105}$ Few reports show the success of in vivo electroporation technique in the nervous system of embryonic mice ${ }^{106}$ that can be used to repair the injured or diseased CNS. In mice brain organotypic slice cultures, both biolistics and electroporation techniques provide better transfer rate and transgene expression than lipotransfection technique ${ }^{107}$ providing the use of nonviral techniques for therapeutic purposes in clinical studies.

Nanotechnology could also be used for the targeted gene delivery. ${ }^{108}$ Research is in progress to use this technology to transfer the gene or drug at particular site which is otherwise impossible in clinical studies. Studies are in progress to use ultrasound as a physical force to introduce transgenes in CNS. ${ }^{109}$

\section{Conclusion}

Genes with good expression ability of therapeutic molecules have significant potential in CNS injury. Many viral vectors are available to deliver therapeutic genes at target site. However, CNS gene delivery remains a challenge. A smart viral delivery vehicle with optimal gene titer could solve the problems associated with CNS therapeutic gene delivery. In vivo studies suggest that recombinant virus along with lentivirus is a better and more promising vehicle for long-term efficient transgene expression in CNS tissues. Other option perhaps is a nonviral, targeted transgenes delivery with CNS compatible biomaterial. This emerges as a viable option for CNS gene delivery and therapeutic expression for use in many neuronal diseases. In the place of in vivo gene delivery, ex vivo gene delivery techniques looks very exciting for the repair of lost tissues after TBI or necrosis in CNS.

\section{Acknowledgments}

Authors acknowledge financial support from the Canadian Institute of Health Research (CIHR) grant to Prakash and University Grants Commission (UGC), India to AK, TDS, and SKS. We would also like to thank technical support from Jasmine Bhathena.

\section{References}

1. Yang K, Clifton GL, Hayes RL. Gene therapy for central nervous system injury: the use of cationic liposomes: an invited review. JNeurotrauma. 1997;14(5):281-297.

2. Zou LL, Huang L, Hayes RL, et al. Liposome-mediated NGF gene transfection following neuronal injury: potential therapeutic applications. Gene Ther. 1999;6(6):994-1005.

3. Hagihara Y, Saitoh Y, Kaneda Y, Kohmura E, Yoshimine T. Widespread gene transfection into the central nervous system of primates. Gene Ther. 2000;7(9):759-763.

4. Brooks AI, Halterman MW, Chadwick CA, et al. Reproducible and efficient murine CNS gene delivery using a microprocessor-controlled injector. J Neurosci Methods. 1998;80(2):137-147.

5. Satoh T, Enokido Y, Kubo T, Yamada M, Hatanaka H. Oxygen toxicity induces apoptosis in neuronal cells. Cell Mol Neurobiol. 1998;18(6):649-666.

6. Zou L, Kumar A, Yuan X, Hannay J, Shine D, Yang K. Improvement of cognitive function and cholinergic neuron transmission by adenovirusmediated nerve growth factor gene transfer in aged rat brain. Mol Ther. 2001;3:S125-S126.

7. Holtzman DM, Sheldon RA, Jaffe W, Cheng Y, Ferriero DM. Nerve growth factor protects the neonatal brain against hypoxic-ischemic injury. Ann Neurol. 1996;39(1):114-122.

8. Mattson MP, Scheff SW. Endogenous neuroprotection factors and traumatic brain injury: mechanisms of action and implications for therapy. J Neurotrauma. 1994;11(1):3-33.

9. Meuli-Simmen C, Liu Y, Yeo TT, et al. Gene expression along the cerebral-spinal axis after regional gene delivery. Hum Gene Ther. 1999;10(16):2689-2700.

10. Lapchak PA, Hefti F. BDNF and NGF treatment in lesioned rats: effects on cholinergic function and weight gain. Neuroreport. 1992;3(5):405-408.

11. Shao L, Ciallella JR, Yan HQ, et al. Differential effects of traumatic brain injury on vesicular acetylcholine transporter and M2 muscarinic receptor mRNA and protein in rat. J Neurotrauma. 1999;16(7):555-566.

12. Guillin O, Demily C, Thibaut F. Brain-derived neurotrophic factor in schizophrenia and its relation with dopamine. Int Rev Neurobiol. 2007;78:377-395.

13. Singh TD, Mizuno K, Kohno T, Nakamura S. BDNF and trkB mRNA expression in neurons of the neonatal mouse barrel field cortex: normal development and plasticity after cauterizing facial vibrissae. Neurochem Res. 1997;22(7):791-797.

14. Monfils MH, Cowansage KK, LeDoux JE. Brain-derived neurotrophic factor: linking fear learning to memory consolidation. Mol Pharmacol. 2007;72(2):235-237.

15. Caldeira MV, Melo CV, Pereira DB, Carvalho RF, Carvalho AL, Duarte CB. BDNF regulates the expression and traffic of NMDA receptors in cultured hippocampal neurons. Mol Cell Neurosci. 2007;35(2):208-219.

16. Singh TD, Basham ME, Nordeen EJ, Nordeen KW. Early sensory and hormonal experience modulate age-related changes in NR2B mRNA within a forebrain region controlling avian vocal learning. J Neurobiol. 2000;44(1):82-94

17. Singh TD, Nordeen EJ, Nordeen KW. Song tutoring triggers CaMKII phosphorylation within a specialized portion of the avian basal ganglia J Neurobiol. 2005;65(2):179-191.

18. Giza CC, Maria NS, Hovda DA. N-methyl-D-aspartate receptor subunit changes after traumatic injury to the developing brain. $J$ Neurotrauma. 2006;23(6):950-961.

19. Kumar A, Zou LL, Yuan XQ, Long Y, Yang K. N-methyl-D-aspartate receptors: Transient loss of NR1/NR2A/NR2B subunits after traumatic brain injury in a rodent model. $J$ Neurosci Res. 2002;67(6):781-786.

20. Qiu YH, Zhao X, Hayes RL, et al. Activation of phosphatidylinositol 3-kinase by brain-derived neurotrophic factor gene transfection in septo-hippocampal cultures. J Neurosci Res. 1998;52(2):192-200. 
21. Kapeller R, Cantley LC. Phosphatidylinositol 3-kinase. Bioessays. 1994;16(8):565-576.

22. Schabitz WR, Steigleder T, Cooper-Kuhn CM, et al. Intravenous brainderived neurotrophic factor enhances poststroke sensorimotor recovery and stimulates neurogenesis. Stroke. 2007;38(7):2165-2172.

23. Li L, Shui QX, Liang K, Ren H. Brain-derived neurotrophic factor rescues neurons from bacterial meningitis. Pediatr Neurol. 2007;36(5):324-329.

24. Xu DG, Crocker SJ, Doucet JP, et al. Elevation of neuronal expression of NAIP reduces ischemic damage in the rat hippocampus. Nat Med. 1997;3(9):997-1004.

25. Susin SA, Lorenzo HK, Zamzami N, et al. Mitochondrial release of caspase-2 and -9 during the apoptotic process. J Exp Med. 1999;189(2):381-394.

26. Shimazaki K, Urabe M, Monahan J, Ozawa K, Kawai N. Adenoassociated virus vector-mediated bcl-2 gene transfer into post-ischemic gerbil brain in vivo: prospects for gene therapy of ischemia-induced neuronal death. Gene Ther. 2000;7(14):1244-1249.

27. Dunnett SB, Bjorklund A. Prospects for new restorative and neuroprotective treatments in Parkinson's disease. Nature. 1999;399(6738): A32-A39.

28. Yamada M, Oligino T, Mata M, Goss JR, Glorioso JC, Fink DJ. Herpes simplex virus vector-mediated expression of Bcl-2 prevents 6-hydroxydopamine-induced degeneration of neurons in the substantia nigra in vivo. Proc Natl Acad Sci U S A. 1999;96(7):4078-4083.

29. Takahashi K, Schwarz E, Ljubetic C, Murray M, Tessler A, Saavedra RA. DNA plasmid that codes for human Bcl-2 gene preserves axotomized Clarke's nucleus neurons and reduces atrophy after spinal cord hemisection in adult rats. J Comp Neurol. 1999;404(2):159-171.

30. Saavedra RA, Murray M, de LS, Tessler A. In vivo neuroprotection of injured CNS neurons by a single injection of a DNA plasmid encoding the Bcl-2 gene. Prog Brain Res. 2000;128:365-372.

31. Cheung NS, Beart PM, Pascoe CJ, John CA, Bernard O. Human Bcl-2 protects against AMPA receptor-mediated apoptosis. $J$ Neurochem. 2000;74(4):1613-1620.

32. Yenari MA, Fink SL, Sun GH, et al. Gene therapy with HSP72 is neuroprotective in rat models of stroke and epilepsy. Ann Neurol. 1998;44(4):584-591.

33. Plumier JC, Krueger AM, Currie RW, Kontoyiannis D, Kollias G, Pagoulatos GN. Transgenic mice expressing the human inducible Hsp70 have hippocampal neurons resistant to ischemic injury. Cell Stress Chaperones. 1997;2(3):162-167.

34. Yang GY, Zhao YJ, Davidson BL, Betz AL. Overexpression of interleukin-1 receptor antagonist in the mouse brain reduces ischemic brain injury. Brain Res. 1997;751(2):181-188.

35. Tuszynski MH, Weidner N, McCormack M, Miller I, Powell H, Conner J. Grafts of genetically modified Schwann cells to the spinal cord: survival, axon growth, and myelination. Cell Transplant. 1998;7(2):187-196.

36. Weidner N, Blesch A, Grill RJ, Tuszynski MH. Nerve growth factor-hypersecreting Schwann cell grafts augment and guide spinal cord axonal growth and remyelinate central nervous system axons in a phenotypically appropriate manner that correlates with expression of L1. J Comp Neurol. 1999;413(4):495-506.

37. Nakahara Y, Gage FH, Tuszynski MH. Grafts of fibroblasts genetically modified to secrete NGF, BDNF, NT-3, or basic FGF elicit differential responses in the adult spinal cord. Cell Transplantation. 1996;5(2):191-204.

38. Grill R, Murai K, Blesch A, Gage FH, Tuszynski MH. Cellular delivery of neurotrophin-3 promotes corticospinal axonal growth and partial functional recovery after spinal cord injury. $J$ Neurosci. 1997;17(14):5560-5572.

39. Ridet JL, Corti O, Pencalet $\mathrm{P}$, et al. Toward autologous ex vivo gene therapy for the central nervous system with human adult astrocytes. Hum Gene Ther. 1999;10(2):271-280.

40. Cao L, Zhao YC, Jiang ZH, et al. Long-term phenotypic correction of rodent hemiparkinsonism by gene therapy using genetically modified myoblasts. Gene Ther. 2000;7(5):445-449.
41. Segovia J, Vergara P, Brenner M. Astrocyte-specific expression of tyrosine hydroxylase after intracerebral gene transfer induces behavioral recovery in experimental parkinsonism. Gene Ther. 1998; 5(12):1650-1655.

42. Liu Y, Himes BT, Tryon B, et al. Intraspinal grafting of fibroblasts genetically modified by recombinant adenoviruses. Neuroreport. 1998;9(6):1075-1079.

43. Taylor RM, Wolfe JH. Decreased lysosomal storage in the adult MPS VII mouse brain in the vicinity of grafts of retroviral vector-corrected fibroblasts secreting high levels of beta-glucuronidase. Nat Med. 1997;3(7):771-774.

44. Wang YL, Omalley BW, Tsai SY, Omalley BW. A regulatory system for use in gene transfer. Proc Natl Acad Sci U S A. 1994;91(17):8180-8184.

45. Heikinheimo O, Haukkamaa M, Lahteenmaki P. Distribution of RU 486 and its demethylated metabolites in humans. J Clin Endocrinol Metab. 1989;68(2):270-275.

46. Abruzzese RV, Godin D, Burcin M, et al. Ligand-dependent regulation of plasmid-based transgene expression in vivo. Hum Gene Ther. 1999;10(9):1499-1507.

47. Burcin MM, Schiedner G, Kochanek S, Tsai SY, O’Malley BW. Adenovirus-mediated regulable target gene expression in vivo. Proc Natl Acad Sci U S A. 1999;96(2):355-360.

48. Oligino T, Ghivizzani S, Wolfe D, et al. Intra-articular delivery of a herpes simplex virus IL-1Ra gene vector reduces inflammation in a rabbit model of arthritis. Gene Ther. 1999;6(10):1713-1720.

49. Ghersa P, Gobert RP, Sattonnet-Roche P, Richards CA, Pich EM, van Huijsduijnen RH. Highly controlled gene expression using combinations of a tissue-specific promoter recombinant adenovirus and a tetracyclineregulatable transcription factor. Gene Ther. 1998;5(9):1213-1220.

50. Blesch A, Uy HS, Diergardt N, Tuszynski MH. Neurite outgrowth can be modulated in vitro using a tetracycline-repressible gene therapy vector expressing human nerve growth factor. J Neurosci Res. 2000;59(3):402-409.

51. Passini MA, Wolfe JH. Widespread gene delivery and structurespecific patterns of expression in the brain after intraventricular injections of neonatal mice with an adeno-associated virus vector. $J$ Virol. 2001;75(24):12382-12392.

52. Burger C, Nguyen FN, Deng J, Mandel RJ. Systemic mannitol-induced hyperosmolality amplifies rAAV2-mediated striatal transduction to a greater extent than local co-infusion. Mol Ther. 2005;11(2):327-331.

53. Nguyen JB, Sanchez-Pernaute R, Cunningham J, Bankiewicz KS. Convection-enhanced delivery of AAV-2 combined with heparin increases TK gene transfer in the rat brain. Neuroreport. 2001; 12(9): 1961-1964.

54. Burton EA, Fink DJ, Glorioso JC. Replication-defective genomic HSV gene therapy vectors: Design, production and CNS applications. Curr Opin Mol Ther. 2005;7(4):326-336.

55. Samaniego LA, Neiderhiser L, DeLuca NA. Persistence and expression of the herpes simplex virus genome in the absence of immediate-early proteins. J Virol. 1998;72(4):3307-3320.

56. Krisky DM, Marconi PC, Oligino TJ, et al. Development of herpes simplex virus replication-defective multigene vectors for combination gene therapy applications. Gene Ther. 1998;5(11):1517-1530.

57. Wolfe D, Goins WF, Yamada M, et al. Engineering herpes simplex virus vectors for CNS applications. Exp Neurol. 1999;159(1):34-46.

58. Wu P, Phillips MI, Bui J, Terwilliger EF. Adeno-associated virus vectormediated transgene integration into neurons and other nondividing cell targets. J Virol. 1998;72(7):5919-5926.

59. Stavropoulos TA, Strathdee CA. An enhanced packaging system for helper-dependent herpes simplex virus vectors. $J$ Virol. 1998;72(9):7137-7143.

60. Zhang X, O'Shea H, Entwisle C, Boursnell M, Efstathiou S, Inglis S. An efficient selection system for packaging herpes simplex virus amplicons. J Gen Virol. 1998;79(Pt 1):125-131.

61. Wang Y, Yu L, Geller AI. Diverse stabilities of expression in the rat brain from different cellular promoters in a helper virus-free herpes simplex virus type 1 vector system. Hum Gene Ther. 1999;10(11):1763-1771. 
62. Goins WF, Lee KA, Cavalcoli JD, et al. Herpes simplex virus type 1 vector-mediated expression of nerve growth factor protects dorsal root ganglion neurons from peroxide toxicity. $J$ Virol. 1999;73(1):519-532.

63. Johnston JC, Gasmi M, Lim LE, et al. Minimum requirements for efficient transduction of dividing and nondividing cells by feline immunodeficiency virus vectors. $J$ Virol. 1999;73(6):4991-5000.

64. Kim VN, Mitrophanous K, Kingsman SM, Kingsman AJ. Minimal requirement for a lentivirus vector based on human immunodeficiency virus type 1. J Virol. 1998;72(1):811-816.

65. Wong LF, Goodhead L, Prat C, Mitrophanous KA, Kingsman SM, Mazarakis ND. Lentivirus-mediated gene transfer to the central nervous system: therapeutic and research applications. Hum Gene Ther. 2006;17(1):1-9.

66. Naldini L, Blomer U, Gage FH, Trono D, Verma IM. Efficient transfer, integration, and sustained long-term expression of the transgene in adult rat brains injected with a lentiviral vector. Proc Natl Acad Sci U S A. 1996;93(21):11382-11388.

67. Geraerts M, Eggermont K, Hernandez-Acosta P, Garcia-Verdugo JM, Baekelandt V, Debyser Z. Lentiviral vectors mediate efficient and stable gene transfer in adult neural stem cells in vivo. Hum Gene Ther. 2006; 17(6):635-650.

68. Watson DJ, Kobinger GP, Passini MA, Wilson JM, Wolfe JH. Targeted transduction patterns in the mouse brain by lentivirus vectors pseudotyped with VSV, Ebola, Mokola, LCMV, or MuLV envelope proteins. Mol Ther. 2002;5(5 Pt 1):528-537.

69. Cepko CL, Ryder E, Austin C, Golden J, Fields-Berry S, Lin J. Lineage analysis using retroviral vectors. Methods. 1998;14(4):393-406.

70. Davidson BL, Stein CS, Heth JA, et al. Recombinant adeno-associated virus type 2, 4, and 5 vectors: transduction of variant cell types and regions in the mammalian central nervous system. Proc Natl Acad Sci US A. 2000;97(7):3428-3432.

71. Burger C, Gorbatyuk OS, Velardo MJ, et al. Recombinant AAV viral vectors pseudotyped with viral capsids from serotypes 1,2 , and 5 display differential efficiency and cell tropism after delivery to different regions of the central nervous system. Mol Ther. 2004;10(2):302-317.

72. Mori S, Wang L, Takeuchi T, Kanda T. Two novel adeno-associated viruses from cynomolgus monkey: pseudotyping characterization of capsid protein. Virology. 2004;330(2):375-383.

73. Bartlett JS, Samulski RJ, McCown TJ. Selective and rapid uptake of adenoassociated virus type 2 in brain. Hum Gene Ther. 1998;9(8):1181-1186.

74. Fu H, Muenzer J, Samulski RJ, et al. Self-complementary adenoassociated virus serotype 2 vector: global distribution and broad dispersion of AAV-mediated transgene expression in mouse brain. Mol Ther 2003;8(6):911-917.

75. Hermens WTJM, Giger RJ, Holtmaat AJGD, Dijkhuizen PA, Houweling DA, Verhaagen J. Transient gene transfer to neurons and glia: Analysis of adenoviral vector performance in the CNS and PNS. J Neurosci Methods. 1997;71(1):85-98.

76. Xiao X, Li J, McCown TJ, Samulski RJ. Gene transfer by adenoassociated virus vectors into the central nervous system. Exp Neurol. 1997;144(1):113-124.

77. Xiao X, Li J, Samulski RJ. Production of high-titer recombinant adenoassociated virus vectors in the absence of helper adenovirus. $J$ Virol. 1998;72(3):2224-2232.

78. Grimm D, Kern A, Rittner K, Kleinschmidt JA. Novel tools for production and purification of recombinant adenoassociated virus vectors. Hum Gene Ther. 1998;9(18):2745-2760.

79. Zolotukhin S, Byrne BJ, Mason E, et al. Recombinant adeno-associated virus purification using novel methods improves infectious titer and yield. Gene Ther. 1999;6(6):973-985.

80. Clark KR, Liu XL, McGrath JP, Johnson PR. Highly purified recombinant adeno-associated virus vectors are biologically active and free of detectable helper and wild-type viruses. Hum Gene Ther. 1999;10(6):1031-1039.

81. Summerford C, Bartlett JS, Samulski RJ. AlphaVbeta5 integrin: a co-receptor for adeno-associated virus type 2 infection. Nat Med. 1999;5(1):78-82.
82. Grimm D, Kern A, Pawlita M, Ferrari FK, Samulski RJ, Kleinschmidt JA. Titration of AAV-2 particles via a novel capsid ELISA: packaging of genomes can limit production of recombinant AAV-2. Gene Ther. 1999;6(7):1322-1330.

83. Lu H, Chen L, Wang J, et al. Complete correction of hemophilia A with adeno-associated viral vectors containing a full-size expression cassette. Hum Gene Ther. 2008;19(6):648-654.

84. Muramatsu S. [Gene therapy for Parkinson's disease]. Brain Nerve. 2007;59(4):425-430.

85. Fan DS, Ogawa M, Fujimoto K, et al. Behavioral recovery in 6-hydroxydopamine-lesioned rats by cotransduction of striatum with tyrosine hydroxylase and aromatic L-amino acid decarboxylase genes using two separate adeno-associated virus vectors. Hum Gene Ther. 1998;9(17):2527-2535.

86. Louboutin JP, Reyes BAS, Agrawal L, Van Bockstaele E, Strayer DS. Strategies for CNS-directed gene delivery: in vivo gene transfer to the brain using SV40-derived vectors. Gene Ther. 2007;14(12): 939-949.

87. Strayer DS, Agrawal L, Cordelier P, et al. Long-term gene expression in dividing and nondividing cells using SV40-derived vectors. Mol Biotechnol. 2006;34(2):257-270.

88. Morral N, Parks RJ, Zhou H, et al. High doses of a helper-dependent adenoviral vector yield supraphysiological levels of alpha1-antitrypsin with negligible toxicity. Hum Gene Ther. 1998;9(18):2709-2716.

89. Morsy MA, Gu M, Motzel S, et al. An adenoviral vector deleted for all viral coding sequences results in enhanced safety and extended expression of a leptin transgene. Proc Natl Acad Sci U S A. 1998;95(14):7866-7871.

90. Cregan SP, MacLaurin J, Gendron TF, et al. Helper-dependent adenovirus vectors: their use as a gene delivery system to neurons. Gene Ther. 2000;7(14):1200-1209.

91. Feng MZ, Jackson WH, Goldman CK, et al. Stable in vivo gene transduction via a novel adenoviral/retroviral chimeric vector. Nature Biotechnol. 1997;15(9):866-870.

92. Johnston KM, Jacoby D, Pechan PA, et al. HSV/AAV hybrid amplicon vectors extend transgene expression in human glioma cells. Hum Gene Ther. 1997;8(3):359-370.

93. Costantini LC, Jacoby DR, Wang S, Fraefel C, Breakefield XO, Isacson $\mathrm{O}$. Gene transfer to the nigrostriatal system by hybrid herpes simplex virus/adeno-associated virus amplicon vectors. Hum Gene Ther. 1999;10(15):2481-2494.

94. Fraefel C, Jacoby DR, Lage C, et al. Gene transfer into hepatocytes mediated by helper virus-free HSV/AAV hybrid vectors. Mol Med. 1997;3(12):813-825.

95. Sena-Esteves M, Saeki Y, Camp SM, Chiocca EA, Breakefield XO. Single-step conversion of cells to retrovirus vector producers with herpes simplex virus-Epstein-Barr virus hybrid amplicons. $J$ Virol. 1999;73(12):10426-10439.

96. Tan BT, Wu L, Berk AJ. An adenovirus-Epstein-Barr virus hybrid vector that stably transforms cultured cells with high efficiency. $J$ Virol. 1999;73(9): 7582-7589.

97. Oehmig A, Fraefel C, Breakefield XO, Ackermann M. Herpes simplex virus type 1 amplicons and their hybrid virus partners, EBV, AAV, and retrovirus. Curr Gene Ther. 2004;4(4):385-408.

98. Recchia A, Parks RJ, Lamartina S et al. Site-specific integration mediated by a hybrid adenovirus/adeno-associated virus vector. Proc Natl Acad Sci U S A. 1999;96(6):2615-2620.

99. Wiesenhofer B, Kaufmann WA, Humpel C. Improved lipid-mediated gene transfer in C6 glioma cells and primary glial cells using FuGene. J Neurosci Methods. 1999;92(1-2):145-152.

100. Wiesenhofer B, Humpel C. Lipid-mediated gene transfer into primary neurons using FuGene: comparison to $\mathrm{C} 6$ glioma cells and primary glia. Exp Neurol. 2000;164(1):38-44.

101. Saeki Y, Matsumoto N, Nakano Y, Mori M, Awai K, Kaneda Y. Development and characterization of cationic liposomes conjugated with HVJ (Sendai virus): reciprocal effect of cationic lipid for in vitro and in vivo gene transfer. Hum Gene Ther. 1997;8(17):2133-2141. 
102. Namiki Y, Takahashi T, Ohno T. Gene transduction for disseminated intraperitoneal tumor using cationic liposomes containing non-histone chromatin proteins: cationic liposomal gene therapy of carcinomatosa. Gene Ther. 1998;5(2):240-246.

103. Kaneda Y, Saeki Y, Morishita R. Gene therapy using HVJ-liposomes: the best of both worlds? Mol Med Today. 1999;5(7):298-303.

104. Drabick JJ, Glasspool-Malone J, Somiari S, King A, Malone RW. Cutaneous transfection and immune responses to intradermal nucleic acid vaccination are significantly enhanced by in vivo electropermeabilization. Mol Ther. 2001;3(2):249-255.

105. Maruyama H, Miyazaki J, Gejyo F. Epidermis-targeted gene transfer using in vivo electroporation. Methods Mol Biol. 2005;289:431-436.

106. Swartz M, Eberhart J, Mastick GS, Krull CE. Sparking new frontiers: using in vivo electroporation for genetic manipulations. Dev Biol. 2001;233(1):13-21.

107. Murphy RC, Messer A. Gene transfer methods for CNS organotypic cultures: a comparison of three nonviral methods. Mol Ther. 2001;3(1):113-121.

108. Jain KK. Nanobiotechnology - Based drug delivery to the central nervous system. Neurodegener Dis. 2007;4(4):287-291.

109. Hynynen K. Ultrasound for drug and gene delivery to the brain. Adv Drug Deliv Rev. 2008;60(10):1209-1217.

110. Bishop KM, Hofer EK, Mehta A, et al. Therapeutic potential of CERE110 (AAV2-NGF): targeted, stable, and sustained NGF delivery and trophic activity on rodent basal forebrain cholinergic neurons. Exp Neurol. 2008;211(2):574-584.

111. Bennett GD, Wlodarczyk B, Calvin JA, Craig JC, Finnell RH. Valproic acid-induced alterations in growth and neurotrophic factor gene expression in murine embryos. Reprod Toxicol. 2000;14(1):1-11.
112. Cernaianu G, Brandmaier P, Scholz G, et al. All-trans retinoic acid arrests neuroblastoma cells in a dormant state. Subsequent nerve growth factor/brain-derived neurotrophic factor treatment adds modest benefit. J Pediatr Surg. 2008;43(7):1284-1294.

113. Kordower JH, Isacson O, Emerich DF. Cellular delivery of trophic factors for the treatment of Huntington's disease: Is neuroprotection possible? Exper Neurol. 1999;159(1):4-20.

114. Podlesniy P, Kichev A, Pedraza C, et al. Pro-NGF from Alzheimer's disease and normal human brain displays distinctive abilities to induce processing and nuclear translocation of intracellular domain of p75NTR and apoptosis. Am J Pathol. 2006;169(1):119-131.

115. Salehi A, Delcroix JD, Swaab DF. Alzheimer's disease and NGF signaling. J Neural Transm. 2004;111(3):323-345.

116. Xiromerisiou G, Hadjigeorgiou GM, Eerola J, et al. BDNF tagging polymorphisms and haplotype analysis in sporadic Parkinson's disease in diverse ethnic groups. Neurosci Lett. 2007;415(1):59-63.

117. Kanthasamy AG, Kitazaw M, Kaul S, et al. Proteolytic activation of proapoptotic kinase PKC delta is regulated by overexpression of bcl-2 implications for oxidative stress and environmental factors in Parkinson's disease. Ann N Y Acad Sci. 2003;1010:683-686.

118. Voloboueva LA, Duan M, Ouyang Y, Emery JF, Stoy C, Giffard RG. Overexpression of mitochondrial Hsp70/Hsp75 protects astrocytes against ischemic injury in vitro. J Cereb Blood Flow Metab. 2008;28(5):1009-1016.

119. Andre R, Lerouet D, Kimber I, Pinteaux E, Rothwell NJ. Regulation of expression of the novel IL-1 receptor family members in the mouse brain. J Neurochem. 2005;95(2):324-330. 